\title{
Tecnologías físicas y sociales
}

Lucas Lavado Mallqui

Universidad San Martín de Porres

Vivimos una época en la cual, por extraño que parezca, muchos individuos bastante cultivados consideran que la verdad no merece ningún respeto especial.

Harry G. Frankfurt: Sobre la Charlatanería (on bullshit) y sobre la verdad.

\section{Resumen}

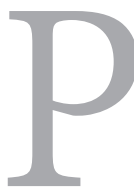

arte de la justificación de aclaración conceptual de la tecnología, ciencia y artefacto, muestra algunos ejemplos de cambio tecnológico expresados en la constante innovación de estos artefactos tecnológicos. En este artículo se asume el enfoque conceptual bungeano y una postura crítica respecto de los negadores de la objetividad y la verdad como de los constructivistas y relativistas.

\section{Palabras clave:}

Tecnología, ciencia, artefacto tecnológico, constructivismo, sociotecnología, verdad y eficiencia.

\section{Introducción}

Por qué es importante estudiar y aclarar el concepto de tecnología en las condiciones actuales? Primero, porque constituye una de las columnas de la política social y el desarrollo. Segundo, porque la vida en el siglo XXI, es una vida instalada en un universo poblado por artefactos tecnológicos. Y tercero, el diseño de proyectos de investigación requiere elucidación conceptual.

La tecnología es un cuerpo de conocimientos que ha surgido de la necesidad de resolver problemas prácticos, como parte de una gama de respuestas que la especie humana ha inventado para hacer frente a los apremios de la vida cotidiana y de los grandes desafíos que conciernen a la humanidad. 
Estamos obligados a entender conceptualmente lo que es la tecnología, diferenciándola de los artefactos tecnológicos tanto físicos como sociales, así como sus relaciones con las ciencias básicas y aplicadas.

\section{La manifestación de los cambios tecnológicos}

Desde inicios de la época moderna, las revoluciones científica y tecnológica fueron tiempos estelares para la humanidad, mucho ha cambiado el sistema mundo debido a los superordenadores, internet y la digitalización. Pensemos en la manera en que Gutemberg trabajaba con los tipos móviles de madera en su imprenta: letra por letra, palabra por palabra, frase por frase y página por página hasta construir un libro tal como lo conocemos. Y pensemos en la manera cómo funcionan hoy las impresoras láser ubicadas en el escritorio de muchos hogares, donde un estudiante puede imprimir su trabajo para la escuela o la universidad. En los millones de los libros, que ya están liberados de pago de derechos de autor que Google ha escaneado. Basta estar conectado a una computadora para diseñar, elaborar y editar nuestro propio libro sin ninguna dificultad. Asistimos a la candente discusión sobre la desaparición de los libros de papel y la puesta en el mercado del libro electrónico.

La puesta en funcionamiento de la PC, el escáner, la impresora 3D instalados en la Galaxia Internet enfrentando el espacio-tiempo, ha posibilitado la reducción de las distancias, de modo que podemos conversar con cualquier persona de cualquier lugar del mundo, iniciar investigaciones interdisciplinares compartidas, entre personas que no tienen que verse cara a cara, o justificar la limitación del idioma. Un mundo nuevo de nativos digitales y analfabetos científicos y tecnológicos. Estamos pues impelidos a diseñar y cultivar nuevos modos de aprender, si no queremos quedar excluidos del sistema cultural global.

Junto con los cambios anteriores, hay otros que son realmente apabullantes, la combinación entre ciencia y tecnología que afecta la propia naturaleza humana. Todos podemos pensar en la prótesis; como los anteojos, los corazones artificiales y los brazos ortopédicos, pero ¿podemos imaginarnos una prótesis cognitiva, es decir implantes en el cerebro para aumentar nuestra memoria y 
nuestra capacidad de respuesta inmediata a las necesidades urgentes de conocimientos? Bueno, estamos en momentos de importancia crucial para enfrentar impactantes cambios para la propia especie humana.

Este desarrollo y progreso de la humanidad, también ha traído nuevos problemas como el deterioro de medio ambiente y la inequitativa distribución de los beneficios y de la felicidad humana. Sus consecuencias están afectando, como nunca antes, las bases mismas de la supervivencia humana. Sumergidos en un mundo artificial que el hombre ha creado, estamos viviendo en un planeta, donde el mismo hombre se ha convertido como dice Jesús Mosterín en el "cáncer de la biosfera". Un mundo que ha quedado atrás para siempre. No tenemos que retroceder mucho, para recordar las máquinas y los teléfonos que ya son objetos de museo. Quizá la crónica del recuadro siguiente le diga algo a un abuelo de hoy y casi nada a los nativos digitales:

\section{Un mundo del pasado que no volverá}

SÁBADO en la noche de un fin de semana cualquiera a mediados de los años 80's. Un adolescente de 18 años sale de su casa para encontrarse con sus amigos y dirigirse en grupo a una fiesta. Las coordinaciones se habían hecho telefónicamente horas antes y no había de qué preocuparse. Al llegar al punto de reunión, no encuentra a su grupo. Espera durante largos minutos hasta que decide comprar varias fichas RIN. Al fin encuentra un teléfono público operativo y saca de su billetera una agenda acordeón donde almacena los teléfonos de las casas de sus amigos. Ya todos habían salido. Vuelve al punto de reunión con la esperanza de que haya sido simplemente un retraso involuntario. Nada. Se resigna y enciende su Walkman para hacer más llevadero su viaje de regreso. Total, es temprano, piensa, tendrá tiempo de pasar ese nivel imposible de Space Invaders en su Atari.

Hoy, ese joven ya es un hombre que bordea los 40 años y recuerda con un buen humor esa anécdota de un tiempo en que la tecnología estaba a punto de vivir una revolución que cambiaría la vida del ser humano: la invención de Internet. En estos días es impensable no estar conectado al mundo ni un solo segundo del día a través de un celular inteligente o cualquier otro dispositivo conectado a esa red mundial de información (Caretas. Lima, 10.12.09, pp. 91-92).

El mundo ha cambiado de un modo que a los muy jóvenes les es difícil maginar el relato anterior. Es como cuando relatásemos pasajes de la época donde 


\section{Teoría}

no existía lápiz, cuaderno de apuntes y libro de texto: aquellas clases de absoluto silencio para que el profesor exponga su tema y los alumnos memoricen lo que dijo el profesor. Es impresionante entender cómo estos artefactos cambiaron la vida para siempre gracias a la tecnología cuyos productos se denominan artefactos.

¿Qué es lo que cambia en la sociedad? La manera de relacionarnos con nuestros semejantes, la forma de comprar y vender, las diferentes formas de trabajar, los lenguajes con los que nos comunicamos, la manera de hacer la guerra, etc. etc. Todo cambia con tal velocidad, que es urgente suponer que las novedades se multiplican cada día. No sabemos lo que nos espera mañana. ¿Pero por qué no nos sorprende tanto las aulas de clase que siguen siendo las mismas de hace 300 años? Debemos trabajar, debemos educarnos y formarnos, debemos vivir para explicar este proceso multidimensional. La telefonía móvil inserto en Internet ha sido factor decisivo de las redes sociales y ha desplazado en cierto modo al popular ordenador personal.

\section{El móvil omnipotente}

Llamar y recibir llamadas es sólo el big bang de la telefonía celular. Apenas dos décadas después de su nacimiento, el móvil se ha alejado de esa causa originaria y compite por sustituir, cada vez con más éxito, al ordenador, la cámara de fotos o de vídeo, la televisión o el GPS del coche. Son los llamados Smartphone o teléfonos inteligentes.

Como certifica la Unión Internacional de Telecomunicaciones (UIT) en su último informe, el celular es la tecnología que más rápidamente se ha adoptado en la historia. A finales de este año, contará con 4,600 millones de abonados. De 2000 a 2008 se ha pasado de una penetración del móvil en el mundo del 12\% al 60\%, mientras que el fijo ha pasado del 16\% al 19\%. La informática tardó cuatro décadas en popularizarse y aún hoy sólo una tercera parte de la humanidad tiene acceso a un ordenador.

$\ldots$

...Lo mejor está aún por venir. Los operadores comienzan a hacer pruebas de laboratorio con LTE (Long Term Evolution), la cuarta generación (4G) celular, con estándar universal, con la que, sólo en su comienzo, alcanzarán velocidades de descarga de hasta 320 Mbps y 80 Mbps en subida. (Ramón Muñoz, El País, 29.12.09, p.24). 
Los retos son frontales, la velocidad del cambio ha sobrepasado las posibilidades de las instituciones educativas. No se trata de clases del profesor, ni solo de la adquisición de libros actualizados, sino del sistema universitario como organización inteligente que ha ingresado a una crisis irreversible. El periodista y profesor Jeff Jarvis (2010) pregunta “¿Quién necesita una universidad cuando tenemos a Google? Todo el conocimiento digital del mundo está disponible en una búsqueda [...] No hay razón para que mis hijos se limiten a asistir a los cursos de una escuela, sino que ahora pueden obtener online cursos del mismísimo MIT y de Stanford” (p. 281). Si este desafío no ha llegado a las mallas curriculares y las clases de nuestras universidades, ya hemos perdido la batalla.

\section{¿Qué futuro nos aguarda?}

Cuando finalizaba el siglo XX los periódicos noticiaban que el ajedrecista campeón mundial, Garry Kasparov, había sido derrotado por la supercomputadora Deep Blue. Tuvo una repercusión mundial, inclusive el mismo ajedrecista creyó que efectivamente jugó con la máquina y perdió. Después de los años transcurridos merece la pena repensar cómo es que ganó la computadora. Las especulaciones y las consecuencias que se extrajeron llevó a sobrevalorar sus posibilidades. La conmoción del ajedrecista ruso fue publicitada y es posible que haya llegado a entender, mucho después, que con quien jugó realmente fue con el equipo de programadores que realimentaban permanentemente el programa.

El profesor Bunge (1997), días después, escribía un artículo periodístico que ponía en evidencia la necesidad de la crítica y la importancia de la epistemología en los currículos. Con su habitual sentido crítico plantea dos tesis: “Tesis 1. Quien derrotó a Kasparov no fue una máquina, sino el equipo de programadores que diseñó el programa Deep Blue. Tesis 2. Las computadoras, aun las programadas de la manera más inteligente, tienen una inteligencia combinatoria antes que creadora" (p. 6). Con lo que queda planteado que quienes vencieron a Kasparov fueron los programadores, aunque esta idea contradiga al profesor Michio Kaku (2014, p.285). Lo que los programadores hicieron es combinar una serie finita de movidas posibles, de modo que la máquina utilizaba inteligencia combinatoria más que imaginación. Con lo que se consolida la nueva 


\section{Teoría}

disciplina denominada inteligencia artificial y la necesidad de estudiar matemáticas, especialmente algoritmos.

Esta nueva rama de la ingeniería le atribuye inteligencia a las máquinas que son capaces de ejecutar algunas operaciones que pueden ser denominadas "inteligentes" y capaces, asimismo, de reconocer órdenes o instrucciones verbales. Encara dos problemas básicos, que como dice el profesor Kaku son "el reconocimiento de patrones y el sentido común" (p. 286). Asociada a otra disciplina denominada robótica, abre horizontes y problemas inéditos de investigación. La lista de disciplinas nuevas también se va haciendo larga y los cambios del mundo del trabajo se hacen cada vez más exigentes en sinergia con la sociedad de la información y del conocimiento.

El ajedrecista David Levy, doctorado en Inteligencia Artificial y escritor especializado en robótica, formula algunas anticipaciones inquietantes respecto de los avances en el campo de la robótica. Prestar atención a lo que dice, será muy ilustrativo para tomar nota sobre cómo están ocurriendo las cosas. Dice con cierta convicción “(n)o tengo duda de que acabará habiendo matrimonios con robots. Y me permito apuntar que se legalizará primero en el Estado de Massachusetts". Pero no se queda allí:

\section{El futuro está muy cerca}

PREGUNTA. Usted dice que en unos años tendremos sexo con robots y nos casaremos con ellos. ¿En qué se basa?

RESPUESTA. No es una opinión superficial sino el producto de años de investigación y del cotejo de fuentes muy variadas. Se lo explicaré brevemente. La inteligencia artificial progresa cada vez más rápido. Mucho más rápido que hace 20 años. Y progresará todavía más rápido en el futuro. En apenas 30 o 40 años los ordenadores serán mucho más poderosos y los científicos podrán crear robots muy similares a las personas. Programas con emociones artificiales que se asemejen a las de los seres humanos. No hay nada que nosotros podamos hacer que un robot no pueda hacer dentro de 30 años.

P. Explíquese.

R. Hoy ni siquiera los ordenadores más poderosos son tan poderosos como el cerebro humano. Según diversos expertos, en unos 12 años los científicos podrán crear un ordenador con el mismo poder que el cerebro humano. Y tardará uno 10 años más 
en crear uno cuya capacidad sea 10,000 veces mayor. Esa es la esencia de mi tesis. A partir de 2040, todo lo que hace de alguien una persona atractiva se podrá reproducir artificialmente, y esto abre la puesta a un futuro incierto.

P. ¿Un futuro que conduce inexorablemente al sexo con robots?

R. Yo no diría inexorablemente, pero no tengo duda de que habrá robots que se parezcan más y más a los seres humanos. Fíjese usted en esta mujer (abre un libro de robótica y aparece una hermosa presentadora). Lo que usted ve es un robot japonés. Es una réplica exacta de una periodista de la televisión nipona. Los creadores reconocen que la perfección de la réplica es relativa y cifran en 10 segundos el tiempo que una persona tarda en darse cuenta de que no es una persona sino un robot. Pues bien, los mismos expertos dicen que dentro de unos años ese tiempo de reacción habrá crecido hasta los 10 minutos. Y poco a poco cada vez más hasta que las diferencias sean imperceptibles (David Levy entrevistado por Eduardo Suárez. El Mundo, 6.9.08)

\section{¿Entonces, qué es tecnología?}

Ahora, la pregunta inevitable: ¿qué es tecnología? La tecnología es un tipo de conocimiento que se ocupan de transformar y controlar los hechos tanto naturales, sociales como socionaturales. Gracias a la tecnología, se ha creado un mundo artificial en el que estamos cómodamente instalados. Como si esto fuera poco, todo el sistema mundo está interconectado, es decir forma sistemas, pequeños, medianos y grandes. Lo nuevo se asoma con el perfil de la ciudad inteligente o smart city.

Se suele establecer diferencias entre técnica y tecnología, aunque muchos autores hoy los utilizan indistintamente. La primera incluye solamente los conocimientos prácticos tradicionales, es decir pre científicos, la tecnología es en cambio un conocimiento práctico que se funda necesariamente en la ciencia contemporánea. Toda la tecnología actual se funda en la ciencia. Hoy no existe tecnología sin base científica.

¿Por qué es importante definir la tecnología y caracterizarla? Porque aclara las ideas y orienta la práctica. Una definición adecuada, permitirá estudiar la tecnología actual con provecho y también encontrar derrotero adecuado para continuar otras búsquedas. 
Tomaremos prestado la definición del filósofo Mario Bunge $(1985,1999)$, quien la define como un sistema compuesto de una 11-tupla, $\mathrm{T}=<\mathrm{C}, \mathrm{S}, \mathrm{D}, \mathrm{G}, \mathrm{F}$, $\mathrm{B}, \mathrm{P}, \mathrm{C}, \mathrm{O}, \mathrm{M}, \mathrm{V}>$, explicitado del modo siguiente:

1. la comunidad profesional $\mathrm{C}$ de $\mathrm{T}$ es un sistema social compuesto por individuos que han sido formados para ejercer una profesión, que siguen una tradición de diseño y evaluación de artefactos de algún tipo, por ejemplo ingenieros informáticos y administradores;

2.S, la sociedad que alberga a $\mathrm{C}$ los apoya o al menos tolera sus actividades profesionales;

3.el dominio D o universo de discurso de T está compuesto exclusivamente (certificadas o putativamente reales), pasadas, presentes o futuras, algunas naturales y otras artificiales;

4.elenfoquegeneralotrasfondofilosóficoGdeTconsistenteena) unaontologíadecosas cambiantes conforme a leyes -en particular recursos naturales y cosas bajo control humano posible-, b) una epistemología realista con un toque de pragmatismo y c) la ética profesional de la utilización de los recursos naturales y humanos (cognitivos en particular);

5. el trasfondo formal $\mathrm{F}$ de una colección de teorías lógicas y matemáticas actualizadas.

6. el trasfondo especifico B de T es una colección de datos, hipótesis y teorías actualizados, razonablemente confirmados (aunque corregibles) acerca de métodos de investigación efectivos y de diseños, normas y planes encontrados en otros campos del conocimiento, en particular en las ciencias y las tecnologías relacionadas con $\mathrm{T}$,

7. la problemática $\mathrm{P}$ de $\mathrm{T}$ consiste exclusivamente en problemas cognitivos y prácticos acerca de los miembros del dominio $\mathrm{D}$, así como en problemas acerca de los otros miembros de la 11-tupla.

8. el fondo de conocimientos $C$ de T es una colección de datos, hipótesis, teorías y métodos actualizados y verificables (aunque no definitivos), así como de diseños, normas y planes compatibles con el trasfondo específico B y obtenidos por algunos miembros de $\mathrm{C}$ en ocasiones anteriores.

9. los objetivos $\mathrm{O}$ de los miembros de Cincluyen inventar artefactos o procesos nuevos, nuevas formas de usar los conocidos, o normas y planes para llevarlos a cabo o para evaluarlos.

10. la metódica $\mathrm{M}$ de $\mathrm{T}$ consiste exclusivamente de procedimientos escrutables (verificables, analizables, criticables) y justificables (explicables), en particular el método científico y el método tecnológico. 
11. Los valores $\mathrm{V}$ de $\mathrm{T}$ consisten en una colección de juicios de valor acerca de cosas y procesos naturales o artificiales -en particular materias primas o productos terminados, recursos humanos, procesos de trabajo, organizaciones sociales y normas.

Quienes realizan investigaciones tecnológicas se encargan de diseñar artefactos de diferentes tipos que siguen un proceso de prueba, para pasar a la fase de la patente y producción industrial. El proceso difiere de la investigación científica.

\section{Las verdades científicas y sus negadores}

Una vez más: en el siglo XXI ya no existen tecnologías ajenas a las ciencias. Todas se fundan en verdades científicas, en hipótesis suficientemente confirmadas. Debido a ello es que en los centros de desarrollo tecnológico avanzado no descuidan las investigaciones básicas en matemática, física, química, biología, sociología, psicología, economía etc. Son los soportes tanto de las tecnologías físicas como de las sociotecnologías.

En las facultades o escuelas de ingeniería, administración, etc., los currículos de asignaturas incluyen ciencia básica y filosofía. Sin un puñado de verdades científicas no sería posible la formación de técnicos, ingenieros, administradores y abogados eficientes. Sin un arsenal de verdades no sería posible diseñar artefactos de tanta precisión y eficientes como por ejemplo las sondas espaciales, los telescopios, los nuevos sistemas de gestión, etc.

La ciencia busca verdades que sirven para orientarnos en el mundo en que vivimos y tomar decisiones importantes de cara a la sociedad. Pero como quiera que todo conocimiento científico y técnico no están desconectados de un enfoque filosófico, es bueno advertir que existen enfoques o posturas que reniegan de la verdad de muchas maneras.

Entre los negadores están los constructivistas y los relativistas. Los primeros cuando relacionan la realidad con el discurso y llevan sus afirmaciones al límite negando la independencia de la realidad respecto del sujeto. Para ellos no existen descubrimientos sino construcciones sociales, según ellos el mundo como tal no existe, lo construimos. Niegan que exploramos y descubrimos el 


\section{Teoría}

mundo con artefactos construidos para tal empresa. Son también negadores de la realidad objetiva.

Los relativistas gnoseológicos, también niegan la existencia de verdades objetivas y transculturales. Sostienen que cada sociedad tiene sus propias maneras de ver y pensar el mundo y las cosas según su punto de vista. Pero, a todas luces, no se puede negar que la ciencia busca verdades, aunque estas sean provisionales. Los científicos nunca dicen que han encontrado verdades científicas definitivas sino aproximaciones.

Los relativistas no creen en las verdades, por ello es que no la buscan. Sin embargo, en muchos lugares del mundo se han establecido comisiones de la verdad, cuando se trata de investigar y juzgar a culpables de violaciones de los derechos humanos. Comisiones que han tenido como objetivo central buscar la verdad y juzgar a los criminales o culpables. Esto ha sido obstaculizado de muchas maneras, unas veces de manera burda.

Sin embargo, felizmente, está pasando de moda minimizar y devaluar la importancia de la verdad a secas. De imponer ideas que socavan la importancia de la verdad de maneras más sutiles, pero igualmente opuestas a la verdad. Existen filósofos de las "medias verdades" y periodistas reputados que, negando la existencia de verdades objetivas, proponen en cambio "una verdad consensual". De este modo los relativistas no piensan que existan argumentos, y exhibición de pruebas, sino negociaciones o "acuerdos" entre las partes. Para ellos no tiene sentido buscar verdades porque no existen. ¿Es así realmente?, ¿no es necesario buscar verdades y defenderlas porque no hacen falta?, ¿Existirían vuelos espaciales y estaciones espaciales sin algunas verdades necesarias para asegurar la empresa y las vidas que ello implica?, ¿son verdades las que revelan en las radiografías de tantos pacientes que buscan curarse?, etc. Es, además, estimulante esta búsqueda.

Teoría hipótesis y diseño

La multiplicidad de teorías incompatibles referentes a un mismo dominio de hechos sólo prueba que la investigación científica no garantiza verdades instantáneas y completas. Pero como lo muestran las pruebas observacionales y experimentales, a menu- 
do damos con hipótesis parcialmente verdaderas. Y, como lo muestra la historia de la ciencia, si una hipótesis es interesante y suficientemente verdadera, estimulará investigaciones que podrán dar como resultado mejores aproximaciones a la verdad. Lo que vale para las hipótesis vale para las teorías y los diseños (las cursivas son nuestras) y resultados experimentales. Al fin y al cabo, el progreso científico no es ilusorio. Y no es concebible sino como el reemplazo de falsedades y de verdades parciales, por verdades más aproximadas, así como el hallazgo de verdades radicalmente nuevas (Bunge, 1998: 198-199).

La ciencia es universal y transcultural, es el tipo de conocimiento indispensable sobre la que se construye proyectos de investigación tecnológica, no solamente en ciencias naturales sino también sociales y socionaturales. No solamente se diseñan y renuevan los ordenadores sino también los artefactos sociotécnicos como son los partidos políticos, las empresas, las universidades, los hospitales, etc. Sin dejar de reconocer que el diseño y puesta en marcha de artefactos sociotécnicos hay más chapuzas que trabajo serio.

¿Existen artefactos sociotécnicos? Sí, existen artefactos sociotécnicos que se diseñan como si se diseñaran motores o puentes, pero que este diseño es mucho más complicado que diseñar puentes, porque se trata de organizaciones compuestas por seres humanos que piensan, sufren y tienen emociones. Para ello necesitamos un puñado de verdades históricas, económicas, sociológicas, psicológicas, etc. Las ciencias sociales y la biología son el soporte para construir artefactos sociotécnicos. Con lo que estamos frente a un tipo de tecnología de lo que no dice nada los manuales universitarios de metodología.

Los constructivismos y relativismos son nocivos porque impiden la investigación que permita diseñar o rediseñar artefactos sociotécnicos como escuelas, partidos, ejércitos, hospitales etc. La desconfianza sistemática sobre la búsqueda de verdades es una manera de limitar, disminuir y dinamitar las investigaciones. Desalienta la investigación científica y tecnológica.

Las ciencias trabajan con hipótesis como su aspecto central y siempre tratan de contrastar estas hipótesis a luz de los hechos, ejemplo la hipótesis provocativa de Martel (2015) de que "[e]l futuro de internet no es global, está enraizado en un territorio" (p. 21). En cambio, quienes hacen investigaciones tecnológicas 
siempre buscar hacer diseños para someterlos a prueba. son ilustrativas las peripecias en el diseño de Wikipedia (Isaacson, 2014). Las ciencias buscan verdades y las tecnologías actuar sobre los hechos buscando el valor de la eficiencia.

\section{La tecnología y los artefactos}

Con frecuencia se suele confundir ciencia aplicada con tecnología y ésta con los artefactos. Tal es la confusión, que algunos intonsos al importar computadores de última generación creen que están contribuyendo con el adelanto tecnológico. Esto ha llevado a reprimir la investigación tecnológica y a utilizar el mismo esquema de investigación para la tesis en derecho, administración y literatura. Les resulta difícil entender que las ciencias tratan de explicar hechos, en tanto que las tecnologías, sobre esta base, transformarlas. Que las ciencias buscan verdades y las tecnologías eficiencias. Otra confusión lamentable es la introducción del neologismo tecnociencia (Bunge, 2015) en los medios universitarios. En el medio peruano, un marco conceptual adecuado para ubicarse en este proceso complejo y poco explorado lo propone Francisco Sagasti (2011).

Finalmente, las ciencias aplicadas también son distintas de las tecnologías, en principio porque son ciencias y buscan verdades, con el añadido importante de su posible utilidad en cualquier campo, ejemplo: el estudio de las plantas de la selva baja peruana pensando en su posible utilidad alimentaria o medicinal.

\section{Conclusiones}

Estamos sumergidos en el mega sistema tecnológico y sus contemporáneo. Estos avances están cambiando la vida de la especie humana en general, está alterando la cotidianidad de las personas que cada día se insertan más a este nuevo sistema. La universidad no está preocupándose por estos cambios, como si no fuese con nosotros. Es urgente, por ello, iniciar un dialogo encaminado a encarar la investigación de amplio espectro enfatizando las sociotecnologías que tienen un considerable retraso en la sociedad moderna. 


\section{Referencias}

Bunge, M. (1985). Seudociencia e ideología. Madrid: Alianza.

Bunge, M. (1997, junio 22). Kasparov vs. IBM ¿Quién ganó? Dominical del Diario El Comercio, p. 6.

Bunge, M. (1998). Elogio de la curiosidad. Buenos Aire: Sudamericana.

Bunge, M. (1999). Buscar la filosofía en las ciencias sociales. México, D.F: Siglo XXI Editores.

Bunge,M.(2015). Evaluandofilosofías.Unaprotesta, unapropuestayrespuestas a cuestiones filosóficas descuidadas. Barcelona: Gedisa.

Isaacson,W.(2014).Losinnovadores.Lahistoriadelosgeniosquecrearoninternet. Barcelona: Penguin Random House.

Jarvis,J.(2010).YGoogle, ¿cómoloharía?Hagasloquehagas,Googleloacabará haciendomejorquetúyademásgratis.¿Estápreparado?Bogotá:Planeta Colombiana.

Kaku, M. (2014). Elfuturo de nuestra mente.El reto científico para entender, mejoraryfortalecernuestramente.Barcelona:Penguin RandomHouse.

Martel, F. (2015). Smart. Internet (s): la investigación. Buenos Aires: Aguilar, Altea, Taurus, Alfaguara.

Sagasti,F.(2011).Ciencia,tecnologíaeinnovación.PolíticasparaAméricaLatina. Lima: Fondo de Cultura Económica del Perú. 